\title{
千岛湖岛屿小型兽类群落分布格局及其影响因素
}

\author{
赵庆洋, 鲍毅新*, 孙波, 张龙龙, 胡知渊
}

（浙江师范大学 生态研究所, 浙江 金华 321004)

\begin{abstract}
摘要: 2007 年秋季和 2008 年春季, 采用夹夜法对千岛湖 14 个岛屿进行小型兽类群落组成调查, 研究其群落 空间分布格局及其影响因素。两季度共布置夹 16800 只, 捕获小型兽类 1037 只, 隶属 2 目 2 科 7 属 11 种。其 中啮齿目（Rodenria）鼠科（Muridae） 9 种, 食虫目（Insectivora）鼠鼠科（Soricdae）2 种。结果显示: 千岛湖 小型兽类群落呈非随机分布格局; 嵌套分析表明, 千岛湖岛屿小型兽类群落呈现极显著嵌套格局, 植物丰富度和 生境专属性两项参数对其嵌套格局的形成具有显著影响, 从而使适应力强的物种能够在多样的环境中生存, 而适 应力弱的物种则局限在特定的环境中。由于取样强度差异会过高地估计嵌套程度, 故在研究中应尽量去除取样强 度的干扰。
\end{abstract}

关键词: 生境片段化; 小型兽类; 嵌套结构; 生境专属性; 千岛湖岛屿

中图分类号: Q142.9; Q958.1 1; Q959.831 文献标识码: A 文章编号: 0254-5853- (2009) 06-0671-08

\section{Community Distribution Pattern and the Affecting Factors of Small Mammals in Qiandao Lake, Zhejiang}

\author{
ZHAO Qing-yang, BAO Yi-xin*, SUN Bo, ZHANG Long-long, HU Zhi-yuan \\ (Institute of Ecology, Zhejiang Normal University, Jinhua, 321004, China)
}

\begin{abstract}
During autumn 2007 and spring 2008, we surveyed 14 islands, with a total of 16800 traps, for small mammals in Qiandao Lake, Zhejiang to determine their community distribution. The captured small mammals belonged to 11 species, 7 genera, 2 families and 2 orders, including 9 species of Murids (Muridae), and 2 species of Soricds (Soricdae). The results showed that the small mammals present a non-random nested pattern in assemblage on the islands. In terms of larger scale perspective, the species of high adaptability were able to adjust to the various environments, while ones of low adaptability only survived in particular circumstances. Plant species richness and habitat specificity of the species were the main ecological factors that influenced the formation of this pattern.
\end{abstract}

Key words: Habitat fragmentation; Small mammals; Nestedness; Habitat specificity; Island of Qiandao Lake

由于人类活动的不断加剧, 在全球范围内生境 片段化程度日趋严重。生境片段化是导致生物多样 性丧失的主要原因之一, 亦成为近年生态学的研究 热点 (Saunder et al, 1991; Andrén et al, 1994; Vitousek et al, 1997; McGarigal et al, 2002）。然而, 长期以来, 针对生境片段化中群落结构的研究大多 关注群落的物种丰度 (species richness) 和多度 ( abundance) (Terborgh et al, 2001; Huai et al, 2007), 而群落结构的另一基本特征一一群落的分布
格局, 一直未能引起重视（Wright et al, 1998）。 群落分布格局的形成是由于竞争等生物作用, 还是由于各物种的随机聚集, 这是一个困扰生态学 家很多年的问题（Weiher \& Keddy，1999）。 Diamond (1975) 提出了一个对岛屿群落理论的发 展有重大影响的论点, 他首先假设动物群落分布的 格局是由竞争决定的, 在这个前提下群落结构都呈 现出非随机分布 (Diamond, 1975)。Coleman (1981) 提出了一个区域内物种随机分布的中位模型（the 
hypothesis of random placement）, 该模型利用观测 所得到的结果来预测该区域物种分布是否随机。

很多研究显示, 在一个岛屿生态系统中, 小岛 屿中出现的物种, 多数也会出现在物种相对丰富的 大岛屿中, 这一非随机分布格局被命名为嵌套结构 ( nestedness ) （Darlington，1957; Amburel \& Temple, 1983; Freemark \& Merriam, 1986; Blake \& Karr, 1987)。Pstterson \& Atmar (1986) 首 次系统地提出了在群落水平构建矩阵, 进行嵌套分 析的方法。之后, 嵌套格局理论在群落生态学领域 被广泛应用于各种栖息地斑块类型中的群落物种 组成和分布格局的研究 (Whitcomb et al, 1981; Pstterson \& Atmar, 1986; Dobson \& Pacala, 1992; Li et al, 1998; Honnay et al, 1999; Fleishman et al, 2002; Fischer \& Lindenmayer, 2005; Greve et al, 2005)。

在自然生态系统中, 小型兽类群落占有重要地 位。它的存在是生态、地理和进化等多种因素共同 作用的结果。小型兽类群落结构的变化, 反映了群 落内的种间关系, 也反映了群落与其外界环境的联 系。目前对小型兽类群落结构的研究多集中于对群 落多样性的研究 (Zhang et al, 1998a， b， 2003）, 而对岛屿栖息地中小型兽类的群落分布格局的研
究少见报道。

千岛湖独特的自然景观与岛屿化栖息地, 加之 近50年的片段化历史, 使千岛湖已经成为研究栖息 地片段化问题的一个重要平台。因此, 本研究在千 岛湖人工湖泊岛屿, 以小型兽类为研究对象, 分析 和验证以下问题：（1）千岛湖岛屿上小型兽类的 分布是否是随机的; (2) 检验千岛湖岛屿小型兽 类群落的分布是否符合嵌套格局; (3) 分析面积、 隔离度和植物物种丰富度等岛屿特征参数, 以及小 型兽类体长、物种的最小分布岛屿面积和生境专属 性等物种生活史特征对嵌套格局的影响。通过对上 述问题的研究与分析，探讨片段化栖息地中的小型 兽类群落分布格局及影响其群落分布格局的因素。

\section{1 研究地概况}

千岛湖位于浙江省西部淳安县境内（图 1), 介 于 $118^{\circ} 34^{\prime} \sim 119^{\circ} 15^{\prime} \mathrm{E}, 29^{\circ} 22^{\prime} \sim 29^{\circ} 50^{\prime} \mathrm{N}$ 之间, 东西 长 $60 \mathrm{~km}$, 南北宽 $50 \mathrm{~km}$, 正常水位 $108 \mathrm{~m}$, 拥有 $573 \mathrm{~km}^{2}$ 水面和 $409 \mathrm{~km}^{2}$ 山场、178 亿蓄水量, 是 1959 年因建造新安江水电站筑坝蓄水而形成的人 工湖。千岛湖上星罗棋布的许多岛屿只是水位上涨 后未完全被水淹没的山峰, 因湖内有 $2500 \mathrm{~m}^{2}$ 以上 岛屿 1078 个而得名。千岛湖地处亚热带季风气

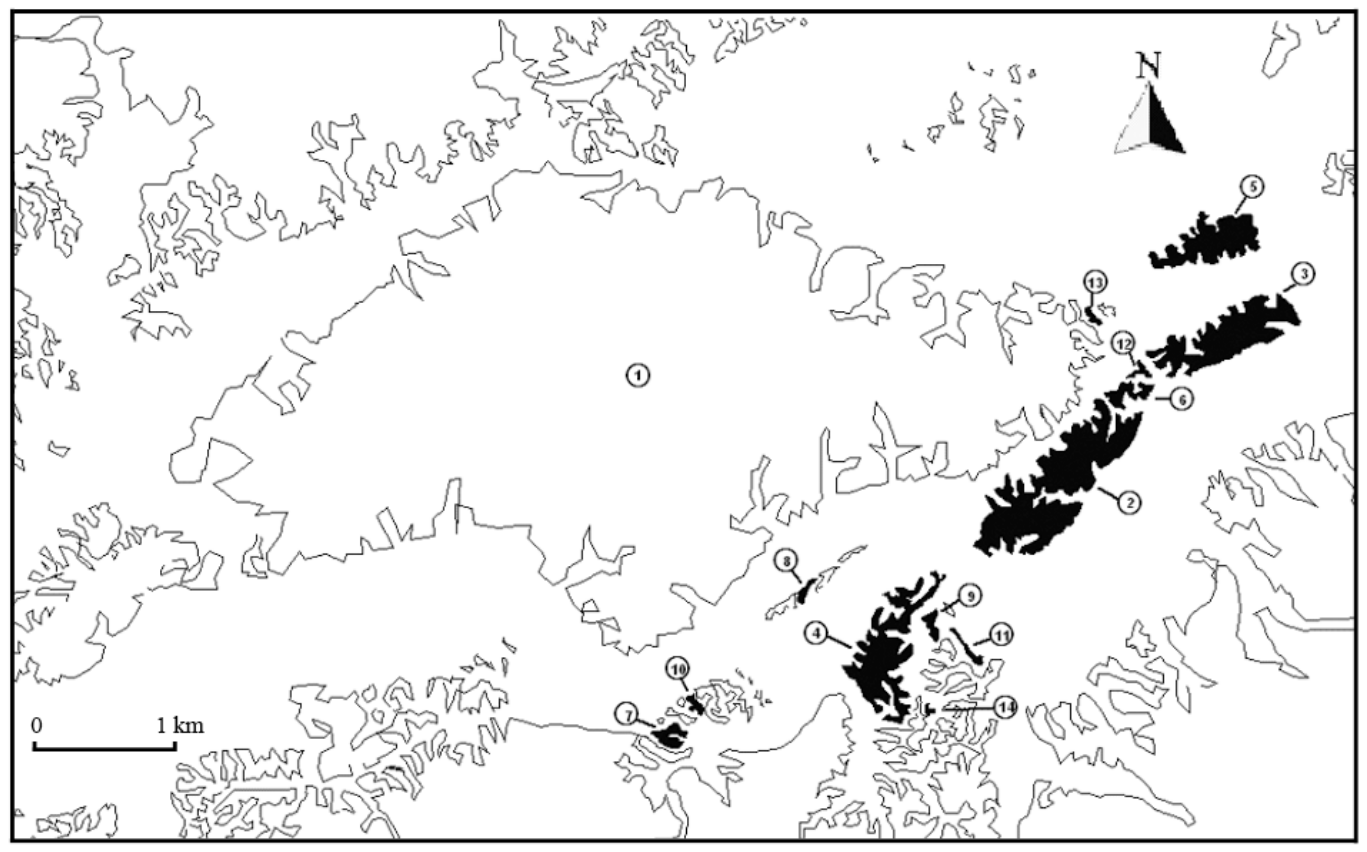

图 1 千岛湖取样岛屿方位图

Fig. 1 Locations of the sampled islands in Qiandao Lake

(1)一(14)为各取样岛屿序号 ( (1)-(14) are the number of the sampled plots)。 
候区的北缘, 气候温暖湿润, 四季分明, 年平均气 温为 $17{ }^{\circ} \mathrm{C}$, 平均降水量 $1430 \mathrm{~mm}$, 年相对湿度 $76 \%$ 。千岛湖区域植被类型属亚热带常绿林, 湖中 岛屿植被以天然次生马尾松 (Pinus massoniana) 林 为主。

\section{2 研究方法}

\section{1 岛屿设置}

2007 年秋季和 2008 年春季, 在千岛湖选取 14 个人为干扰少的岛屿作为取样岛屿, 各岛屿的方位 图见图 1, 特征参数见表 1 。

\section{2 小型兽类种类调查}

根据Terborgh et al (2001) 的研究方法结合研 究区域的实际情况, 对千岛湖的岛屿依据面积 $(S)$ 划分为 3 种类型: 大型岛屿 $\left(S>30 \mathrm{hm}^{2}\right)$ 、中型岛 屿 $\left(2 \mathrm{hm}^{2}<S \leq 30 \mathrm{hm}^{2}\right)$ 、小型岛屿 $\left(0.01 \mathrm{hm}^{2} \leq S \leq 2\right.$ $\mathrm{hm}^{2}$ ) (表 1)。在取样岛屿中放置鼠铗, 以花生米为 诱㱗, 夹距 $5 \mathrm{~m}$ 。根据岛屿面积大小确定置夹数量,
每个大型岛置夹 900 只, 中型岛置夹 600 只, 小型 岛置夹 300 只（表 1)。对于生境类型较多的大型岛 屿, 布夹前, 首先分析该岛的生境类型, 确保鼠夹 在每种生境都有分布; 对于小型岛屿, 布夹贯穿整 岛。当晚下夹, 次日清晨收夹。对各取样岛屿所捕 捉的鼠类分别进行外形测量、分类 (Zhuge， 1990; Wang, 2003) 和常规解剖, 记录所栖息的生境类型。 并计算各物种的体长、物种的最小分布岛屿面积和 生境专属性。其中, 体长为捕获物种样本体长的平 均值 $(\mathrm{mm})$; 物种的最小分布岛屿面积为有该物 种捕获的最小岛屿的面积 $\left(\mathrm{hm}^{2}\right)$; 生境专属性的 计算参照（Zhang et al，2008）的划分标准，将千 岛湖小型兽类栖息地划分成针叶林、阔叶林、针阔 混交林、竹林、灌木、杂草和农田 7 个类型。如果 某种小型兽类只在 1 种类型的栖息地被捕获, 则得 1 分, 为高专属性种类; 如果某种小型兽类在全部 7 种类型的栖息地中都被捕获，则得 7 分，为低专 属性种类。

表 1 取样岛屿的特征参数

Tab. 1 The traits of the sampled islands in Qiandao Lake

\begin{tabular}{|c|c|c|c|c|c|c|c|}
\hline $\begin{array}{l}\text { 岛屿序号 } \\
\text { Number } \\
\text { of islands }\end{array}$ & 岛屿 Island & $\begin{array}{l}\text { 面积 } \\
\text { Area } \\
\left(\mathrm{hm}^{2}\right)\end{array}$ & $\begin{array}{l}\text { 距最近陆地距离 } \\
\text { Distance to the } \\
\text { nearest mainland } \\
\text { (m) }\end{array}$ & $\begin{array}{c}\text { 距最近大岛距离 } \\
\text { Distance to the nearest } \\
\text { bigger island } \\
(\mathrm{m})\end{array}$ & $\begin{array}{c}\text { 植物丰富度 } \\
\text { Plant species } \\
\text { richness }\end{array}$ & $\begin{array}{l}\text { 岛屿 } \\
\text { 等级 } \\
\text { Class }\end{array}$ & $\begin{array}{l}\text { 置夹数（只） } \\
\text { Number } \\
\text { of mousetrap }\end{array}$ \\
\hline 1 & 界首 Jieshou & 1289.23 & 897 & 897 & 198 & $\mathrm{~L}$ & 900 \\
\hline 2 & 大畈龙 Dafanlong & 109.03 & 965 & 132 & 86 & $\mathrm{~L}$ & 900 \\
\hline 3 & 高仙阁 Gaoxiange & 55.08 & 954 & 506 & 59 & $\mathrm{~L}$ & 900 \\
\hline 4 & 坞石畈 Wushifan & 46.37 & 730 & 155 & 51 & $\mathrm{~L}$ & 900 \\
\hline 5 & 东门 Dongmen & 32.29 & 1937 & 471 & 57 & $\mathrm{~L}$ & 900 \\
\hline 6 & 茶坞岭-2 Chawuling-2 & 12.02 & 1158 & 64 & 65 & M & 600 \\
\hline 7 & 竹林坞 Zhulinwu & 9.12 & 24.5 & 855 & 35 & M & 600 \\
\hline 8 & 中岛 Zhongdao & 2.9 & 1785 & 497 & 85 & M & 600 \\
\hline 9 & 灯塔-1 Dengta-1 & 2.83 & 1238 & 155 & 86 & M & 600 \\
\hline 10 & 桥坞坪 Qiaowuping & 1.67 & 570 & 12 & 80 & $\mathrm{~S}$ & 300 \\
\hline 11 & 灯塔-2 Dengta-2 & 1.54 & 711 & 73 & 88 & $\mathrm{~S}$ & 300 \\
\hline 12 & 茶坞岭-1 Chawuling-1 & 1.08 & 1271 & 33 & 43 & $\mathrm{~S}$ & 300 \\
\hline 13 & 拦网头 Lanwangtou & 1.01 & 2104 & 41 & 36 & $\mathrm{~S}$ & 300 \\
\hline 14 & 竹丝坞 Zhusiwu & 0.97 & 597 & 63 & 30 & $\mathrm{~S}$ & 300 \\
\hline
\end{tabular}

L: 大型岛 (large island); M: 中型岛 (moderate island); S: 小型岛 (small island)。

为确定所得到的物种能真实地反映取样岛屿 原本存在的物种，而不是受到随机取样或者偶然入 侵行为的影响是非常重要的。为此, 用以下计算公 式（Aguirre，2003）计算两个季度的物种流动率 $T$ (species turnover rate) :

$$
T=(J+E) /\left(S_{1}+S_{2}\right)
$$

其中, $J$ 是在春季出现而没有在夏季出现的物种数, $E$ 是在秋季出现而没有在春季出现的物种数, $S_{1}$ 和 $S_{2}$ 是分别在两个季度中均出现的物种数。 $T$ 值介于 0 
(物种在岛屿间无流动) 和 1 (物种在岛屿间完全 流动）之间。

\section{3 分析方法}

2.3.1 Random placement 中位模型的检验 用 Random placement模型 (即在一个区域内物种随机 分布的中位模型）测定岛上的物种是否随机分布。 根据Random Placement的模型计算在面积为 $a$ 的第 $k$ 个岛屿上的物种期望值 $S_{a}$ :

$$
\begin{gathered}
S_{a}=S-\sum_{i=1}^{s}(1-\alpha)^{n i} \\
\alpha=a / A
\end{gathered}
$$

$S$ 表示所有 $k$ 个研究岛上的物种的总数, $\alpha$ 是一个 面积为 $a$ 的岛屿的相对面积, $A$ 是所有研究岛屿的 总面积, $n i$ 表示在面积为 $a$ 的第 $k$ 个岛屿上第 $i$ 个 物种的个体数 $(i=1,2, \ldots \ldots, S)$ 。

通过用岛屿的相对面积 $\alpha_{k}$ 的观察值 $S_{k}$ （在第 $k$ 个 岛上物种数量), 我们能知道观察点 $\left(\alpha_{k}, S_{k}\right)$ 与 预测得到的种-面积关系曲线的关系。如果假设正 确, 观察点在 $S_{a}$ 图上一定是随机分布, $S_{a}$ 是根据公 式 (2) 计算所得。

2.3.2 嵌套程度的量化 为了确定嵌套的程度, 需 要根据物种在斑块上的出现情况, 以 1 或 0 表示“出 现”或“不出现”构建矩阵。在本研究中, 采用基于矩 阵温度 (matrix temperature) 计算的方法

( BINMATNEST) ( Rodriguez-Girones \& Santamaria，2006）来量化嵌套程度。 “BINMATNEST" 会对输入的矩阵进行最大化排列, 并计算矩阵温度 $T$ 。 $T=0^{\circ} \mathrm{C}$ 表示完全嵌套, $T=100^{\circ} \mathrm{C}$ 表示完全随机。与其他软件相比, “BINMATNEST" 使用了一种更为健全的计算方法, 能有效解决在计 算过程中不能做到 $T$ 值最低的问题。分析时 BINMATNEST 提供 3 种 null modal 对结果进行显 著性检验, 其中 null modal 3 在研究中被证实能有 效控制被动取样对嵌套显著性的影响（Rodriguez-

Girones \& Santamaria, 2006; Moore \& Swihart, 2007)。

2.3.3 嵌套程度的决定因素 经过 BINMATNEST 计算后得到的物种和岛屿的序列称为嵌套序列 (nested ranking), 可以用来与一些可能与之相关 的因素进行比较, 从而可以确定引起嵌套的因素。 本研究使用 Spearman 序列相关性分析 (spearman's rank correlation) 的方法对嵌套结构的影响因素进行 分析。利用嵌套序列中岛屿的序列和物种的序列分 别与物种的生境特征参数与物种的生活史参数进 行相关性分析, 如果相关性明显, 说明分析因素对 嵌套格局的形成有显著影响（Schouten，2007）。

2.3.4 取样强度对嵌套程度的影响 取样强度的 不同会直接影响到取样的结果 (Rich, 1998; Dennis, 1999）, 为了确定取样强度对取样结果的影响, 通 过计算各岛屿的取样强度 (布夹数 $/ \mathrm{hm}^{2}$ ), 然后依 据取样强度的大小，去掉 $5 \%$ 取样强度高和低的岛 屿, 从而过滤掉高取样强度和低取样强度的岛屿。

所涉及的数据处理及差异性分析均在统计软 件SPSS 13.0中进行, $P \leq 0.05$ 为显著差异, $P \leq 0.01$ 为 极显著差异, $P>0.05$ 为差异不显著。

\section{3 结 果}

\section{1 取样结果}

14 个取样岛屿, 共置鼠夹 16800 只, 捕获小 型兽类 1037 只, 隶属 2 目 11 种, 其中啮齿目 9 种, 食虫目 2 种, 各岛屿捕获物种个体数见表 2 。各物 种生活史特征中体长为 $62 \sim 240 \mathrm{~mm}$; 物种的最小分 布岛屿面积为 $0.97 \sim 1289.23 \mathrm{hm}^{2}$; 生境专属性为 1 7（表 3）。

\subsection{Random placement 模型预测结果}

通过Random placement 模型（公式 2）计算的 各岛屿物种数期望值 $S_{a}$ 与相对面积的对数值 $\ln (\alpha)$ 的关系如图 2。从中可以看到, 所有岛屿的观察值 （observed value）都与所期望的 $S_{a}$ 值有显差异，因 而可以判定各岛屿上的物种并不是随机分布的。

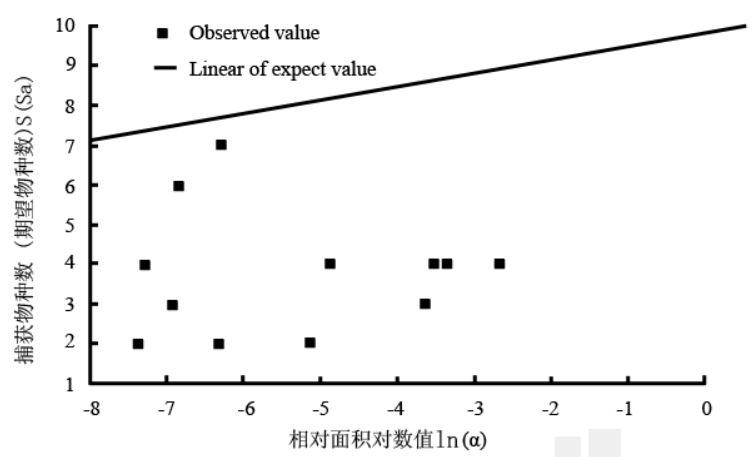

图 2 实际调查结果同 Random placement 模型 预测结果比较

Fig. 2 Compare of observed data with consequences of the hypothesis of random placement

一: 预测值 $S_{a}$; : 实际调查结果。

The solid curve ( - ) is expected number of species, and the observed values are shown as "ø". 
表 2 千岛湖各取样岛屿捕获小型兽类个体数

Tab. 2 Individual number of small mammals capture in Qiandao Lake

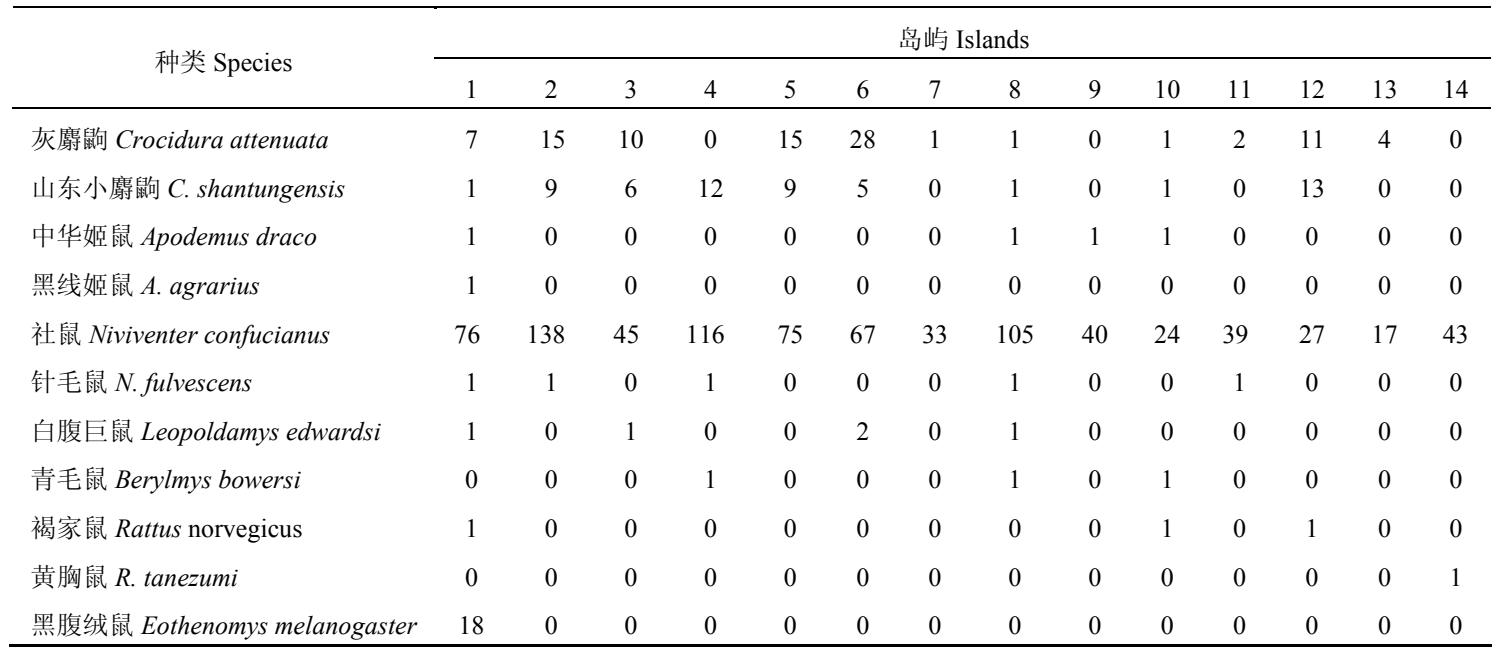

表 3 千岛湖小型兽类生活史特征参数

Tab. 3 Life-history traits of small mammals species in Qiandao Lake

\begin{tabular}{|c|c|c|c|}
\hline 物种 Species & $\begin{array}{c}\text { 体长 } \\
\text { Body length } \\
(\mathrm{mm})\end{array}$ & $\begin{array}{c}\text { 物种的最小分布岛屿面积 } \\
\text { Area of minimal distributed } \\
\text { island }\left(\mathrm{hm}^{2}\right)\end{array}$ & $\begin{array}{c}\text { 生境专属性 } \\
\text { Value of habitat } \\
\text { specificity }\end{array}$ \\
\hline 灰膺鼠 Crocidura attenuata & 77 & 1.01 & 6 \\
\hline 山东小麾鼠 C. shantungensis & 62 & 1.08 & 6 \\
\hline 中华姬鼠 Apodemus draco & 84 & 1.67 & 4 \\
\hline 黑线姬鼠 A. agrarius & 93 & 1289.23 & 1 \\
\hline 社鼠 Niviventer confucianus & 124 & 0.97 & 7 \\
\hline 针毛鼠 N. fulvescens & 141 & 1.54 & 4 \\
\hline 白腹巨鼠 Leopoldamys edwardsi & 219 & 2.9 & 4 \\
\hline 青毛鼠 Berylmys bowersi & 240 & 1.67 & 3 \\
\hline 褐家鼠 Rattus norvegicus & 174 & 1.08 & 2 \\
\hline 黄胸鼠 R. tanezumi & 164 & 0.97 & 1 \\
\hline 黑腹线鼠 Eothenomys melanogaster & 98 & 1289.23 & 4 \\
\hline
\end{tabular}

表 4 千岛湖小型兽类最大化排列后的嵌套矩阵

Tab. 4 Maximally nested matrix for small mammals on studied islands in Qiandao Lake

\begin{tabular}{|c|c|c|c|c|c|c|c|c|c|c|c|c|c|c|}
\hline \multirow{2}{*}{ 种类 Species } & \multicolumn{14}{|c|}{ 岛屿 Islands } \\
\hline & 1 & 8 & 10 & 12 & 6 & 3 & 2 & 4 & 11 & 9 & 5 & 13 & 7 & 14 \\
\hline 社鼠 Niviventer confucianus & 1 & 1 & 1 & 1 & 1 & 1 & 1 & 1 & 1 & 1 & 1 & 1 & 1 & 1 \\
\hline 灰㑗鼠 Crocidura attenuata & 1 & 1 & 1 & 1 & 1 & 1 & 1 & 0 & 1 & 0 & 1 & 1 & 1 & 0 \\
\hline 山东小塵鼠 C. shantungensis & 1 & 1 & 1 & 1 & 1 & 1 & 1 & 1 & 0 & 0 & 1 & 0 & 0 & 0 \\
\hline 中华姬鼠 Apodemus draco & 1 & 1 & 1 & 0 & 0 & 0 & 0 & 0 & 0 & 1 & 0 & 0 & 0 & 0 \\
\hline 针毛鼠 Niviventer fulvescens & 1 & 1 & 0 & 0 & 0 & 0 & 1 & 1 & 1 & 0 & 0 & 0 & 0 & 0 \\
\hline 白腹巨鼠 Leopoldamys edwardsi & 1 & 1 & 0 & 0 & 1 & 1 & 0 & 0 & 0 & 0 & 0 & 0 & 0 & 0 \\
\hline 青毛鼠 Berylmys bowersi & 0 & 1 & 1 & 0 & 0 & 0 & 0 & 1 & 0 & 0 & 0 & 0 & 0 & 0 \\
\hline 褐家鼠 Rattus norvegicus & 1 & 0 & 1 & 1 & 0 & 0 & 0 & 0 & 0 & 0 & 0 & 0 & 0 & 0 \\
\hline 黑腹线鼠 Eothenomys melanogaster & 1 & 0 & 0 & 0 & 0 & 0 & 0 & 0 & 0 & 0 & 0 & 0 & 0 & 0 \\
\hline 黑线姬鼠 A. agrarius & 1 & 0 & 0 & 0 & 0 & 0 & 0 & 0 & 0 & 0 & 0 & 0 & 0 & 0 \\
\hline 黄胸鼠 R. tanezumi & 0 & 0 & 0 & 0 & 0 & 0 & 0 & 0 & 0 & 0 & 0 & 0 & 0 & 1 \\
\hline
\end{tabular}

“1”(阴影) : “存在”; “0”=“不存在” ( “1”: “occurrence”; “0”：“no occurrence” )。 
表 5 千岛湖小型兽类嵌套分析结果

Tab. 5 Results of the nestedness analysis for small mammals on studied islands in Qiandao Lake

\begin{tabular}{|c|c|c|c|c|}
\hline 项目 Item & $\begin{array}{c}\text { 物种数 } \\
\text { Number of species }\end{array}$ & $\begin{array}{l}\text { 岛屿数 } \\
\text { Number of the island }\end{array}$ & $\begin{array}{l}\text { 填充度 } \\
\text { Fill }(\%)\end{array}$ & $\begin{array}{c}\text { 系统温度 } \\
\text { System temperature }\left({ }^{\circ} \mathrm{C}\right)\end{array}$ \\
\hline 所有岛屿 All islands & 11 & 14 & 36 & $9.94^{* * *}$ \\
\hline $\begin{array}{l}\text { 去除 5\%取样强度的岛屿 } \\
\text { Upper and lower } 5 \text { th percentile excluded }\end{array}$ & 8 & 12 & 34 & $15.14^{*}$ \\
\hline
\end{tabular}

表 6 矩阵按各因素排序后与 BINMATNEST 最大化序列间的 Spearman 序列相关性结果

Tab. 6 Results of Spearman's rank correlations between ranking order after packing to maximal nestedness using BINMATNWST and order after rearranging the matrix

\begin{tabular}{|c|c|c|c|c|c|c|c|}
\hline \multirow{2}{*}{ 项目 Item } & \multicolumn{4}{|c|}{ 岛屿特征参数 Traits of the island } & \multicolumn{3}{|c|}{ 生活史特征参数 Life-history traits } \\
\hline & Area & DNM & DNI & PSR & $\mathrm{BL}$ & AMDI & VHS \\
\hline 小型兽类 Small mammals & -0.389 & -0.099 & -0.077 & $-0.585^{*}$ & 0.418 & 0.339 & $-0.916^{* *}$ \\
\hline
\end{tabular}

DNM: 距最近陆地距离 Distance to the nearest mainland; DNI: 距最近大岛距离 Distance to the nearest bigger island; PSR: 植物丰富度 Plant species richness; BL: 体长 Body length; AMDI: 物种的最小分布岛屿面积 Area of minimal distributed island; VHS: 生境专属性 Value of habitat specificity。

\section{3 嵌套分析结果}

矩阵经 BIN 最大化排列后的布局如表 4, 进行 嵌套分析得到的系统温度 $T=9.94^{\circ} \mathrm{C}$, 采用 null modal 3 进行显著性检验, 呈极显著嵌套（表 5 ）。 因此, 千岛湖区小型兽类整体上呈现嵌套格局。

Spearman 序列相关性分析显示岛屿特征参数 中依据植物丰富度排列成的序列与嵌套序列有较 强相关（植物丰富度： $r=-0.585, P<0.05$ ），而岛 屿面积 $(r=-0.389, P>0.05)$ 和反映隔离度的距最 近大岛距离 $(r=-0.077, P>0.05)$ 、距最近陆地距 离 $(r=-0.099, P>0.05) 3$ 项参数未显示出显著的 相关性。物种生活史参数中依据生境专属性排列成 的序列与嵌套序列有较强的相关 $(r=-0.916, P<$ $0.01)$, 而物种的最小分布岛屿面积 $(r=-0.339, P$ $>0.05)$ 和体长参数 $(r=0.418, P>0.05)$ 未显示出 显著相关性。因而, 植物丰富度和生境专属性是对 千岛湖小型兽类嵌套格局贡献最大的参数 (表 6 )。 当过滤掉 $5 \%$ 取样强度较高和较低的岛屿后, 得到 的嵌套温度明显升高 $(T=15.14)$ 而嵌套的显著程度 也降低（表 5）, 这表明取样强度的不同会导致过 高的估计系统的嵌套程度, 但需要指出的是, 最终 的结果仍然显示显著嵌套 $(P<0.05)$ 。

\section{4 讨 论}

通过公式（1）计算得到两个季度所有岛屿间 的物种流通率很低 $(T=0.1)$, 而每个岛屿间得到的
流通率也较低（ $T=0.29$, 平均值）。因而我们可以 确信本研究中所得到的取样结果能真实地反映各 岛屿上原有物种的存在格局。但是需要指出的是: 部分相邻岛屿间的距离较近, 而某些小型兽类又具 有短距离游泳的能力，故不排除有些物种在各岛间 流动的情况, 但长距离涉水对小型兽类而言是极为 困难的，因而一般情况下水对小型兽类在各岛屿间 的流动具有极其明显的限制作用, 否则得到的 $T$ 值 应该会非常高。

以往的研究显示, 多数物种偏爱某一类型的生 境，而生境类型在岛屿间的分布并不是随机的，而 是呈现一种嵌套的分布结构，生境结构的嵌套分布 最终导致物种分布的嵌套结构（Simberloff \& Martin, 1991; Calmé \& Desrochers，1999）。本 项研究显示, 千岛湖岛屿小型兽类群落呈现非随机 分布的格局，用 BINMATNESS 检验后呈现极显著 嵌套格局。对其分布格局的影响因素进行分析, 结 果显示栖息地特征参数中的植物丰富度和生活史 特征参数中的生境专属性对嵌套格局的形成有显 著贡献。植物物种丰富度高的栖息地，由于能为小 型兽类提供更多的食物来源、繁殖场所和隐蔽条件 而倾向于包含更多的种类 (Calmé \& Desrochers, 1999），由岛屿间植物物种丰富度差异造成的对物 种的不同限制, 可能使小型兽类群落在一定程度上 呈现嵌套结构。物种对栖息地适应能力的差异会使 生境专属性低的种类在较多的岛屿上有分布, 如社 
鼠; 而生境专属性高的种类只能在较少的岛屿上分 布, 如黄胸鼠和小家鼠。人工蓄水形成岛屿时, 生 境专属性低的物种可以在多数岛屿上存活, 而物种 丰富度高的岛屿就倾向于留住更多种类的物种。因 而, 本研究结果更趋向于支持生境嵌套假说。

本研究也显示, 取样强度的差异会导致对嵌套 程度的过高估计。被动取样假说强调了嵌套结构形 成的方法学因素, 多度不同的物种, 即便是随机分 布的, 在不同的取样面积下, 同样可以产生物种组 合的嵌套结构。这种嵌套结构完全是由取样产生 的, 与自然界中物种的真实分布状况无关。然而这 种理论是片面的, 因为嵌套结构是一种客观存在的 格局 (Willianms, 1995), 在实际研究中我们要做 的是尽量避免取样效应的干扰 (Calmé \& Desrochers，1999）。

对经过BINMATNEST排列后的矩阵进行分 析, 可以发现物种的存在数据与不存在数据大致相 遇在矩阵的左下角到右上角的对角线附近, 这条对 角线称为边界线 (Liu et al, 2002)。边界线有着重 要意义, 标志该区系的分布极限。超过它, 则种群 的预期是零, 即超过该线物种不存在。我们还能预 计分布最广的物种 (如社鼠) 在包含物种最多的岛 屿（如 1 号岛屿）上具有最大的存活能力, 这种存 活概率朝着物种相对较少的岛屿而下降直到边界 线, 边界线上的矩阵元素意味着相应的岛屿上分布 的种群正面临着最大的灭绝风险。

嵌套结构在保护生物学上具有特殊意义 (Pstterson, 1987; Worthen, 1996), 为自然保护区 的最优化设计提供了新的视角, 从而也重新引发了 SLOSS (single large or several small) 争论。SLOSS 问题的核心是: 一个大的保护区与数个总面积相同 的小保护区, 哪一个更有保护价值? 嵌套结构给予 保护生物学家看待SLOSS问题一个新的视角: 群落 组成。在完全的嵌套结构下, 每一个小岛屿所包含 的物种仅仅是大岛屿的物种组成的子集, 因而数个 小岛屿的物种总和不可能超过单个的大岛屿，也就

\section{参考文献:}

Aguirre LF, Lens L, Damme R, Matthysen E. 2003. Consistency and variation in the bat assemblages inhabiting two forest islands within a neotropical savanna in Bolivia [J]. J Trop Ecol, 19(4): 367-374.

Ambuel B, Temple SA. 1983. Area dependent changes in the bird communities and vegetation of southern Wisconsin forests [J]. Ecology, 64 (5): 1057-1068.
是说, 嵌套结构支持单个大岛屿的保护价值。在现 实的自然界中, 完全的嵌套结构很少存在

（Boecklen，1997）。近来的一些研究也显示, 即 便是岛屿群落的组成存在着显著的嵌套结构, 数个 小岛屿仍可以比相同面积的单个大岛屿含有更多 的物种数 (Beckon, 1993; Cook, 1995; Skaggs \& Boecklen, 1996）。本研究也显示, 有些小型兽类 物种只在小岛屿种出现, 如: 黄胸鼠仅在最小面积 的岛屿（14号岛屿）中出现（表3）。因而, 嵌套 结构不能彻底地解决SLOSS问题, 因为SLOSS本身 就存在着问题, SLOSS仅仅考虑生境的面积, 而忽 略了生境的其他因素。凭单一的因素来判断生境与 物种的保护价值是不科学的。

嵌套性分析仅仅基于种的“存在”与“不存在”数 据。尽管这限制了嵌套性的潜在应用, 但这也恰恰 是它独特的优势（Willianms，1995），因为即使是 最基本的生态调查也会取得一组物种在某时某地 共同出现这样的数据, 这对很多调查不充分的地区 来说, 这样的数据恰恰是我们能够利用的最好的数 据。

综上所述，千岛湖岛屿小型兽类群落分布格局 受到植物丰富度和生境专属性两因素影响, 两因素 的共同作用使适应力强的物种能够在多样的环境 中生存, 而适应力弱的物种则局限在特定的环境 中, 从而在整体上产生一种嵌套格局 (Wright et al, 1998; Schouten et al，2007）。由于在自然生态系统 中, 小型兽类群落占有重要地位, 小型兽类群落结 构的变化既反映了群落内不同物种之间的关系，同 时也反映了群落与其外界环境的联系, 因而本研究 结果可以为千岛湖地区其他物种群落格局研究提 供依据。

致谢: 本文岛屿特征参数数据来源于浙江大学 丁平教授的千岛湖研究小组, 浙江大学王彦平博士 和浙江师范大学颉志刚博士在数据分析上给予大 力帮助, 特此致谢。
Andrén H. 1994. Can one use nested subset pattern to reject the random sample hypotheses? Examples from boreal bird communities [J]. Oikos, 70(3): 489-491.

Beckon N. 1993. The effect of insularity on the diversity of land birds in the Fiji Islands: implications for refuge design [J]. Oecologia, 94(3): 318-329. 
Blake JG, Karr JR. 1987. Breeding birds of isolated woodlots: area and habitat relationships [J]. Ecology, 68(6): 1724-1734.

Boecklen WJ. 1997. Nestedness, biogeographic theory, and the design of nature reserves [J]. Oecologia, 112(1): 123-142.

Calmé S, Desrochers A. 1999. Nested bird and micro-habitat assemblages in a peatland archipelago [J]. Oecologia, 118(3): 361-370.

Coleman BD. 1981. On random placement and species-area relation [J]. Mathenat Biosci,54: 191-215.

Cook RR. 1995. The relationship between nested subsets, habitat subdivision and species diversity [J]. Oecologia, 101(2): 204-210.

Dennis RL, Spark TH, Harvey PB. 1999. Bias in butterfly distribution maps: the effects of sampling effort[J]. J Insect Conserv, 3(1): 33-42.

Diamond JM. 1975. Assembly of Species Communities. Ecology and Ecolution of Communities[M]. Cambridge MA: Harvard University Press, 342-444.

Dobson AP, Pacala SW. 1992. The parasites of Anolis lizards the northern Lesser Antilles II. The structure of the parasite community [J]. Oecologia, 92: 118-125.

Fischer J, Lindenmayer DB. 2005. Nestedness in fragmented landscapes: a case study on birds, arboreal marsupials and lizards [J]. J Biogeogr, 32(10): 1737-1750.

Fleishman E, Betrus CJ, Blair RB, Nally RM, Murphy DD. 2002. Nestedness analysis and conservation planning: the importance of place, environment, and life history across taxonomic groups [J]. Oecologia, 133(1): 78-89.

Freemark KE, Merriam HG. 1986. Importance of area and habitat heterogeneity to bird assemblages in temperate forest fragments [J]. Biol Consev, 36(2): 115-141.

Greve M, Gremmen NM, Gaston KJ, Chown SL. 2005. Nestedness of Southern Ocean Island biotas : ecological perspectives on a biogeographical conundrum [J]. J Biogeogr, 32(1): 155-168.

Honnay O, Hermy M, Choppin P. 1999. Nested plant communities in deciduous forest fragments: species relaxation or nested habitats $[\mathrm{J}]$ ? Oikos, 84(1): 119-129.

Huai HY, Wei WH, Zhang, YL. 2007. The characteristics of Achnatherum splendens community a long the Qinghai Tibet railway [J]. Acta Ecologica Sinica, 27(2):497-503. [淮虎银, 魏万红, 张镱锂. 2007. 青 藏铁路沿线温性草原区茂芨草(Achnatherum splendens)群落特征. 生态学报, 27(2): 497-503.]

Liu CR, Ma KP, Chen LZ. 2002. Nestedness: methods, mechanisms and implications for biological conservation [J]. Acta Phytoecol Sin, 26: 68-72. [刘灿然, 马克平, 陈灵芝. 2002. 嵌套性: 研究方法、形成机 制及其对生物保护的意义. 植物生态学报, 26: 68-72.]

Li YM, Niemelä J, Li DM. 1998. Nested distribution of amphibians in the Zhoushan archipelago, China: Can selective extinction cause nested subsets of species [J]? Oecologia, 113(4): 557-564.

McGarigal K, Cushman SA. 2002. Comparative evaluation of experimental approaches to the study of habitat fragmentation effects [J]. Ecol Appl, 12(2): 335-345.

Moore JE, Swihart RK. 2007. Toward ecologically explicit null models of nestedness [J]. Oecologia, 152(4): 763-777.

Patterson BD, Atmar W. 1986. Nested subsets and the structure of insular mammalian faunas and archipelagos [J]. Biol J Linnean Soc, 28(1-2): $65-82$.

Patterson BD. 1987. The principle of nested subsets and its implications for biological conservation [J]. Biol Conserv, 1(4): 323-34.

Rich TC. 1998. Squaring the circles-bias in distribution maps [J]. Bri Wildl, 9: $213-219$

Rodriguez-Girones MA, Santamaria L. 2006. A new algorithm to calculate the nestedness temperature of presence-absence matrices $[\mathrm{J}] . \quad J$
Biogeogr, 33(5): 924-935.

Saunders DA, Hobbs RJ, Margules CR. 1991. Biological consequences of ecosystem fragmentation: a review [J]. Conserv Biol, 5(1): 18-30.

Schouten MA, Verweij PA, Barendregt A, Kleukers RJM, Ruiter PC. 2007. Nested assemblages of Orthoptera species in the Netherlands: The importance of habitat features and life-history traits [J]. J Biogeogr, 34(11): 1938-1946.

Simberloff D, Martin J. 1991. Nestedness of insular avifaunas: simple summary statistics masking complex species pattern [J]. Ornis Fennica, 68: $178-192$.

Skaggs RW, Boecklen WJ. 1996. Extinctions of montane mammals reconsidered: putting a global-warming scenario on ice $[\mathrm{J}]$. Biodiver Conserv, 5(6): 759-778.

Terborgh J, Lopez L, Nuñez P, Rao M, Shahabuddin G, Orihuela G, Rivero s M, Ascanio R, Adler GH, Lambert TD, Balbas L. 2001. Ecological meltdown in predator free forest fragments [J]. Science, 294(5548) 1923-1926.

Vitousek PM, Mooney J, Lubchenco JM. 1997. Human domination of earth's ecosystems [J]. Science, 277: 494-499.

Wang YX. 2003. A complete checklist of mammal species and subspecies in china. A taxonomic and geographic reference [M]. Beijing: China Forestry Press, 1-223. [王应祥. 2003. 中国哺乳动物种和亚种分类名 录与分布大全. 北京: 中国林业出社, 1-223.]

Weiher E, Keddy P. 1999. Ecological assembly rules: perpectives, advances, retreats [M]. Cambridge UK: Cambridge University press.

Whitcomb RF, Robbins CS, Lynch JF, Whitcomb BL, Klimkiewicz MK, Bystrak D. 1981.Effects of forest fragmentation on avifauna of the eastern deciduous forest[M]// Burgess RL, Sharpe DM. Forest Island Dynamics in Man-Dominated Landscapes. New York: Springer-Ver-lag, 123-205.

Willianms MR. 1995. An extreme-value function model of the species incidence and species-area relationships [J]. Ecology,76(8): 2607-2616.

Worthen WB. 1996. Community composition and nested subset analyses: Basic descriptors for community ecology [J]. Oikos, 76(3): 417-426.

Wright DH, Patterson BD, Mikkelson GM, Cutler A, Atmar W. 1998. A comparative analysis of nested subset patterns of species composition [J]. Oecologia, 113(1): 1-20.

Zhang DM, Jang T, Mahmut, Anwar, Wang JM. 1998a. The community structure and its change of rodents in Alataw Pass [J]. Acta Theriol Sin 18(2): 154-155. [张大铭, 姜 涛, 马合木提, 艾尼瓦尔, 王建明. 1998a. 阿拉山口啮齿动物群落结构及其变化. 兽类学报, 18(2): 154-155.]

Zhang DM, Anwar, Jang T, Jian YL. 1998b. Analysis of the rodent community diversity and species variation in the Junggar Basin [J] .Chn Biodiver, 6(2): 92-98. [张大铭, 艾尼瓦尔, 姜 涛, 寒友里. 1998b. 准噶尔盆地啮齿动物群落多样性与物种变化的分析. 生物 多样性, 6(2): 92-98.]

Zhang JC, Wang YP, Jiang PP, Li P, Yu MJ, Ding P. 2008. Nested analysis of passeriform bird assemblages in the Thousand Island Lake region $[\mathrm{J}]$. Chn Biodiver, 16(4): 321-331. [张竞成, 王彦平, 蒋萍萍, 李 鹏, 于明坚, 丁 平. 2008. 千岛湖雀形目鸟类群落嵌套结构分析. 生 物多样性, 16(4): 321-331.]

Zhang MW, Wang KR, Wang Y, Li B. 2003. Species diversity of rodent communities in the Dongting Lake area [J]. Acta Ecol Sin, 23(11): 2260-2269. [张美文, 王凯荣，王 勇，李 波. 2003. 洞庭湖区鼠类 群落的物种多样性分析. 生态学报, 23(11): 2260-2269.]

Zhuge Y. 1990. Zhejiang Fauan (Mammals) [M]. Hangzhou: Zhejiang Technology Press. [诸葛阳. 1990. 浙江动物志(兽类). 杭州: 浙江科 技出版社.] 